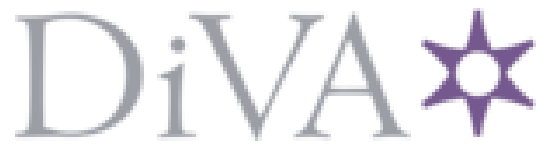

http://www.diva-portal.org

\title{
Postprint
}

This is the accepted version of a paper presented at European Microwave Conference 2017.

Citation for the original published paper:

Svedin, J., Malmqvist, R., Beuerle, B., Shah, U., Oberhammer, J. (2017)

A 230-300 GHz Low-Loss Micromachined Waveguide Hybrid Coupler.

In: Proceedings of the 47th European Microwave Conference, Nuremberg, October

$8-13,2017$

N.B. When citing this work, cite the original published paper.

Permanent link to this version:

http://urn.kb.se/resolve?urn=urn:nbn:se:kth:diva-216956 


\section{A 230-300 GHz Low-Loss Micromachined Waveguide Hybrid Coupler}

\author{
Jan Svedin, Robert Malmqvist \\ Department of Radar Systems \\ Swedish Defence Research Agency (FOI) \\ 58330 Linköping, Sweden \\ jan.svedin@foi.se
}

\author{
Bernhard Beuerle, Umer Shah, Joachim Oberhammer \\ Micro and Nano Systems, School of Electrical Engineering \\ Royal Institute of Technology (KTH) \\ 10044 Stockholm, Sweden \\ joachim.oberhammer@ee.kth.se
}

\begin{abstract}
A silicon micromachined waveguide hybrid coupler for $\mathrm{J}$-band (220-325 GHz) applications is presented. The coupling and phase shifting functions are realised using a $90^{\circ}$ ridged waveguide phase shifter, a joint waveguide section and a port extension. The hybrid coupler is fabricated using a micromachined waveguide technology employing a double $\mathrm{H}$ plane split that results in low losses. For the design band 230-300 GHz, initial VNA measurements indicate coupling coefficients of $-3.2 \pm 0.4 \mathrm{~dB}$, amplitude imbalance better than $-0.5 /+0.3 \mathrm{~dB}$, phase imbalance better than $-14^{\circ} / 11^{\circ}$ and an input reflection coefficient below $-14 \mathrm{~dB}$. To the best of our knowledge, this is the first time a low-loss micromachined hybrid coupler of this type is characterised within this frequency range.
\end{abstract}

Keywords-Micromachining, micromachined waveguide, waveguide coupler, millimeter-wave circuits, submillimeter-wave (sub-MMW), hybrid coupler

\section{INTRODUCTION}

Recent advances in MMIC technology have made development of communication and radar systems operating well into the submillimeter-wave (sub-MMW) spectrum a reality. For wireless communication and active/passive sensing applications such systems have the potential to meet the increasing demand for higher bandwidths. For short-distance radar applications such as security scanning, millimeter-wave and submillimeter-wave systems make high-resolution imaging possible, also at safe stand-off distances.

For a complete sub-MMW system many additional required components, such as low-loss couplers, resonators and antennas, cannot easily be integrated on an MMIC chip in a cost-efficient way. To this date, many of these waveguide components are still manufactured in mechanical workshops using CNC milling and manual assembly, based on split-block designs. The cost for a system built in this way is very high due to the serial nature of the process and the attainable accuracy is insufficient in many cases, in particular for frequencies into the submillimeter-wave region. Micromachining offers a significantly better accuracy and flexibility compared to CNC milling. As it is a highly parallel process, fabrication tolerances are very accurate and micromachining offers the potential for low-cost volume production.

Hybrid and directional couplers are important components in both communication and radar system front-ends. Design, manufacturing and characterization of micromachined couplers have previously been reported up to at least WR-1.5 band [15]. A micromachined hybrid coupler with a measured coupling factor of $-3.4 \pm 0.4 \mathrm{~dB}$ at $85-100 \mathrm{GHz}$ (16\% relative bandwidth) was presented in [1]. More recent experimental studies on micromachined $3-\mathrm{dB}$ and $10-\mathrm{dB}$ directional couplers made at higher frequencies indicate insertion losses in the order of 1-4 $\mathrm{dB}$ at 200-750 GHz. In this paper we report on the design, manufacturing and characterization of a J-band $(220-325 \mathrm{GHz})$ hybrid coupler (3-dB) manufactured using a silicon micromachining waveguide technology developed at KTH [6] with two H-plane splits that enable reduced waveguide losses.

\section{DeSIGN OF HYBRID COUPLER}

The topology used for the J-band coupler is shown in Fig. 1. The coupler has four ports: $\mathrm{P} 1(B), \mathrm{P} 2(\Delta), \mathrm{P} 3\left(\sum\right)$ and $\mathrm{P} 4(A)$. The four ports are connected via short waveguide sections of cross-sectional dimensions $b \times a$ to a joint waveguide section of dimensions $d \times a \times g$. The waveguide section joining P1 to the joint waveguide is a $90^{\circ}$ ridged waveguide phase shifter (using symmetrical inductive irises). The ridge dimensions are $f \times a \times e$ and the ridge-to-ridge distance is $c$. The thickness of the thin wall used to separate the two upper and the two lower waveguides in the figure is $h$. A waveguide extension of length $x$ is added at port P4.

The ideal scattering matrix associated with this coupler,

$$
\boldsymbol{S}=\frac{1}{\sqrt{2}}\left[\begin{array}{rccc}
0 & -1 & i & 0 \\
-1 & 0 & 0 & 1 \\
i & 0 & 0 & i \\
0 & 1 & i & 0
\end{array}\right]
$$

shows its potential use as a power combiner, or as a $3-\mathrm{dB}$ power divider, with achievable phase differences of $0^{\circ}, \pm 90^{\circ}$ and $180^{\circ}$. The design reported in this paper was optimized for use as a $180^{\circ} 3-\mathrm{dB}$ power divider with $\mathrm{P} 2$ as the input and $(\mathrm{P} 4$, $\mathrm{P} 1)$ as the output ports. For this usage scenario (1) gives the following relations between outgoing ( $\mathrm{A}$ and $\mathrm{B}$ ) and incident $\left(\Delta\right.$ and $\sum$ ) waves:

$$
\begin{aligned}
A & =\frac{1}{\sqrt{2}}\left(\Delta+i \sum\right) \\
B & =\frac{1}{\sqrt{2}}\left(-\Delta+i \sum\right)
\end{aligned}
$$


from which the desired $3-\mathrm{dB} 0^{\circ}$ and $180^{\circ}$ power division is obtained by terminating port $\mathrm{P} 3\left(\sum=0\right)$.

An initial design of the J-band hybrid coupler was first made based on a previously reported approach used for a Wband design [1]. This design was then optimized for the desired frequency band using HFSS Optimetrics to obtain a final design for manufacturing. The used goal function involved minimizing $\left|S_{i i}\right|^{2}$ and $\left|S_{12}+S_{42}\right|^{2}$. The following optimized dimensions were obtained and subsequently used for manufacturing: $a=285 \mu \mathrm{m}, b=864 \mu \mathrm{m}, c=478 \mu \mathrm{m}, d=1484$ $\mu \mathrm{m}, e=105 \mu \mathrm{m}, f=52 \mu \mathrm{m}, g=1043 \mu \mathrm{m}, h=105 \mu \mathrm{m}$ and $x=$ $259 \mu \mathrm{m}$ (also see Sect. III).

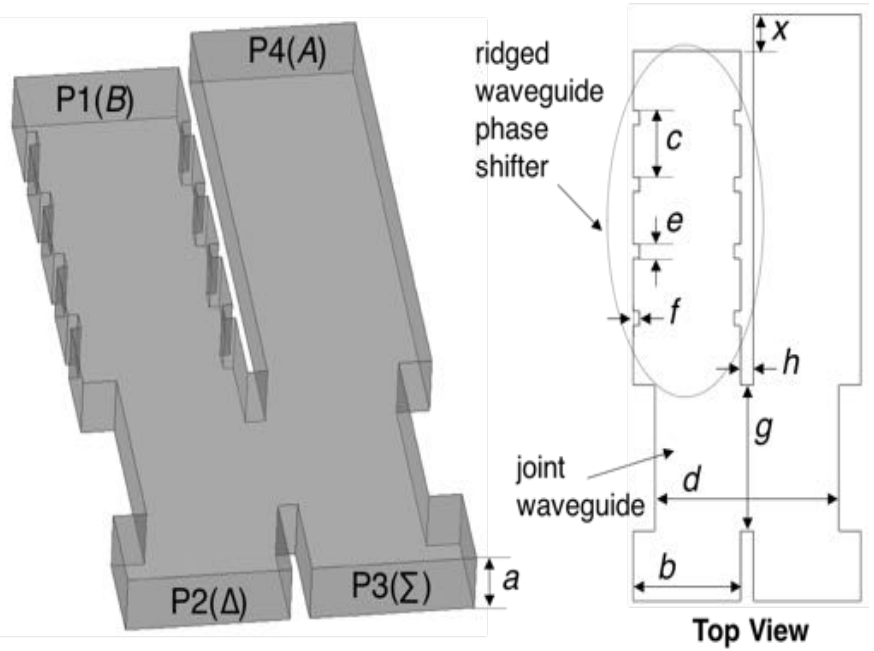

Fig. 1. Topology of the J-band silicon micromachined hybrid coupler.

\section{FABRICATION AND ASSEMBLY}

The J-band hybrid coupler was fabricated in a low-loss micromachined waveguide technology developed at KTH [6], consisting of a silicon-wafer triple stack, i.e. comprising two $\mathrm{H}$-plane splits. The central wafer is $285 \mu \mathrm{m}$ thick which results in a $66 \%$ waveguide height as compared the standard height for WR-3.4 of $432 \mu \mathrm{m}$. The reduced height should only result in minor increase of waveguide losses, which is compensated by the better surface roughness control on the sidewalls for reduced etching depth. The central wafer is etched by deepreactive ion etching, using a silicon dioxide mask. The wafers are metallized by gold sputtering of $1 \mu \mathrm{m}$ and the assembly happens on chip scale by thermo-compression bonding in a tailor-made chip-bonding chamber.

This double H-plane split provides a waveguide concept with near-ideal surface roughness of the bottom and the roof of the waveguide, as only the sidewalls are affected by the silicon etching. This comprises a significant advantage over single $\mathrm{H}$ plane split micromachined waveguides as for instance employed in [8], which is a major reason for the low loss of the coupler. 0 shows a scanning-electron microscope picture of the coupler before bonding to the waveguide bottom.

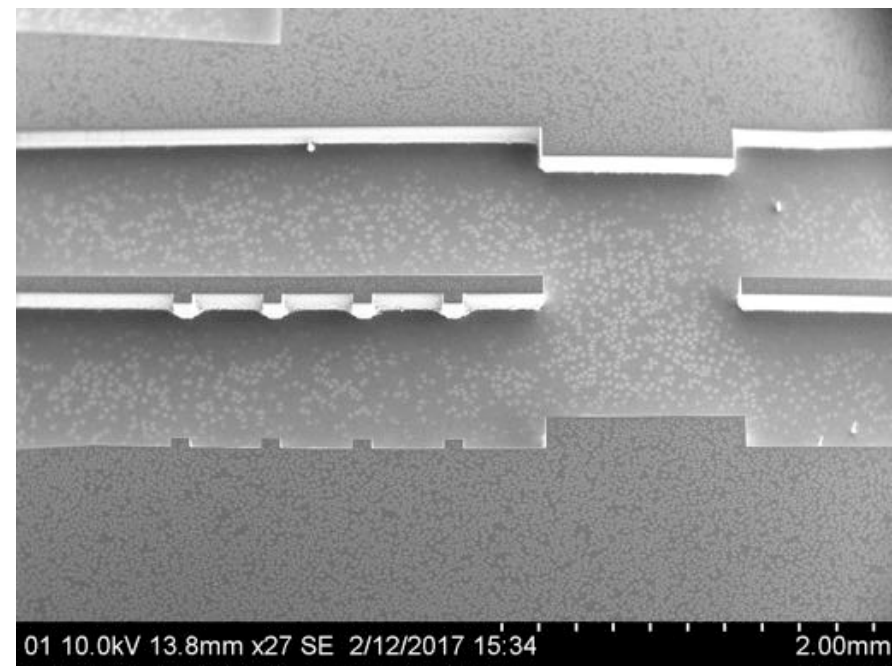

Fig. 2. Scanning electron microscope picture of coupler chip before metallization and final assembly.

\section{MEASUREMENTS}

The measurements were taken using a Rohde\&Schwarz ZVA24 with two ZC330 TxRx extenders. A two-tier TRL calibration was carried out, with the first level using a standard calibration kit and the second level comprising a micromachined calibration kit implemented on the same chip containing the coupler. The micromachined TRL calibration kit allows for de-embedding to reference planes located inside the micromachined rectangular waveguides, i.e., directly adjacent to the coupler ports shown in Fig. 1. A special software was written for remote-controlling the VNA and for showing the de-embedded results in real time.

Since the coupler is a four-port device but only two TxRx modules were available, the remaining two ports had to be terminated by loads. This was facilitated by tapered micromachined absorbers directly integrated in the coupler chip, which have a return loss of at least $15 \mathrm{~dB}$ for the whole band, and are described in [8]. For measuring all port combinations, five different two-port test circuits were manufactured to characterize the different parameters of the coupler. The micromachined couplers for measuring the different port parameters can be considered as nearly identical, due to the high accuracy and device uniformity of micromachining, providing fabrication tolerances of only a few $\mu \mathrm{m}$. The main uncertainty for this multi-device characterization is the variation of the return loss of the micromachined absorbers from device to device, which is less than $2 \mathrm{~dB}$ [8].

Fig. 3 shows some of the measured (solid lines) and simulated (dotted lines) S-parameters for the coupler relevant for the use as a $3-\mathrm{dB} 180^{\circ}$ power divider. Initial measurements indicate coupling coefficients $\left(S_{12}, S_{42}\right)$ of $-3.2 \pm 0.4 \mathrm{~dB}$ and input reflection $\left(S_{22}\right)$ and isolation $\left(S_{32}\right)$ coefficients of $<-14$ $\mathrm{dB}$, for the frequency range 230 to $300 \mathrm{GHz}$. The absolute level of the coupling coefficients is remarkably close to the ideal value of $3 \mathrm{~dB}$, as is also their uniformity over the large bandwidth of the coupler. This is more clearly illustrated in Fig. 4 which also shows the measured quantity $S_{12}+S_{42}$, which 
was used as one part of the goal function optimizing the design, and that should ideally vanish.

The measured amplitude and phase imbalances are plotted in Fig. 5. The amplitude imbalance is found to be better than $0.5 /+0.3 \mathrm{~dB}$. The phase imbalance is found to be better than $14^{\circ} /+11^{\circ}$, which is $7.7 \%$ of $180^{\circ}$. The measurements indicate a performance of the J-band coupler, which is similar to the $\mathrm{W}$ band design [1], despite being at $\sim 3$ times higher frequencies, with only a slightly higher return loss.

The simulated S-parameters show a good agreement with measurements but with a shift upwards in frequency. It is interesting to note that this shift is similar to what was obtained for the W-band design in [1]. The reason for this shift is presently not known. It should be pointed out that our simulations assume ideal matched loads for the ports, whereas the measurements were obtained using the non-ideal micromachined absorbers as terminations for two of the ports in each test circuit.

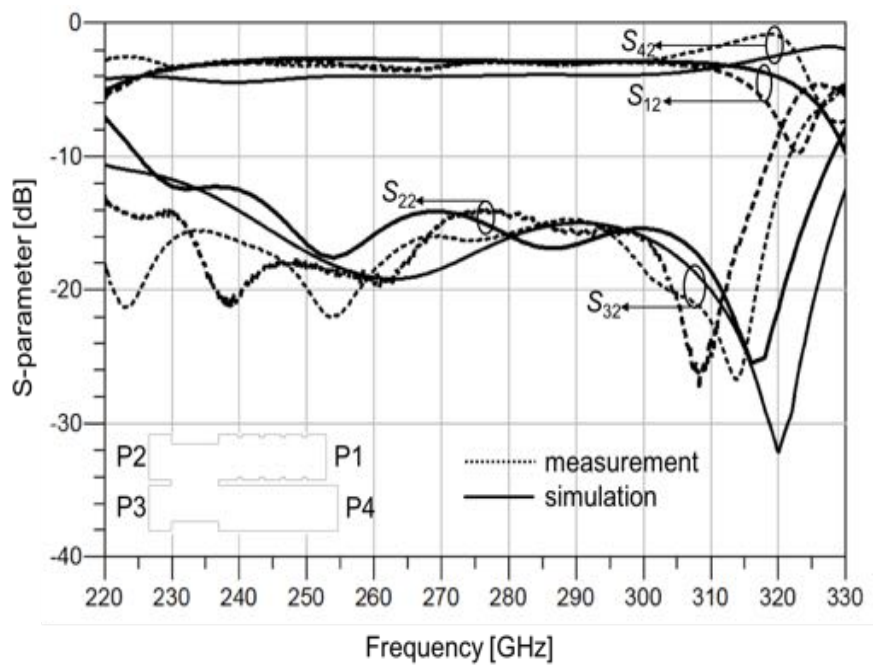

Fig. 3. Measured and simulated magnitudes of input reflection $\left(S_{22}\right)$, coupling $\left(S_{12}, S_{42}\right)$ and isolation $\left(S_{32}\right)$ for the J-band micromachined waveguide hybrid coupler.

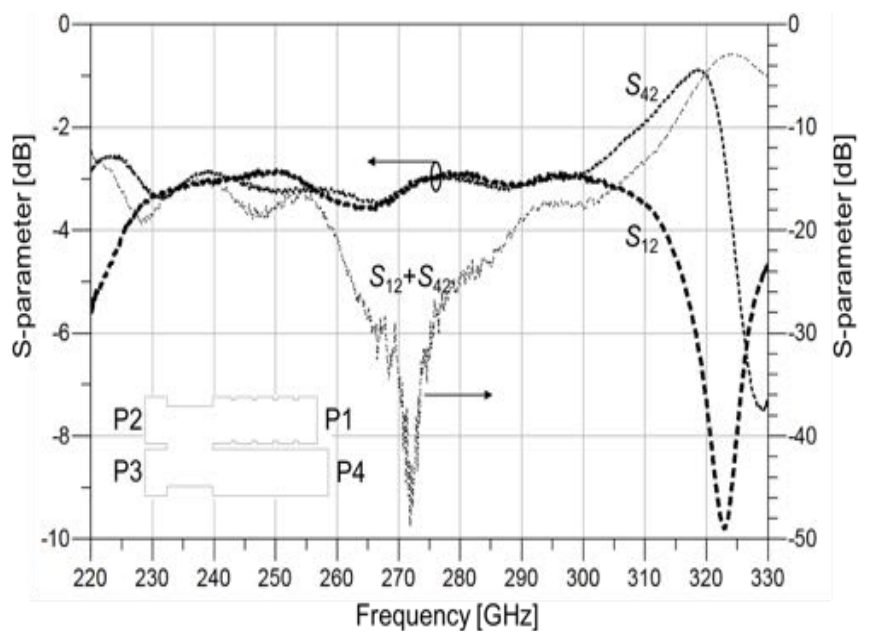

Fig. 4. Measured magnitudes of coupling coefficients and summed output $\left(S_{12}+S_{42}\right)$ for the J-band micromachined waveguide hybrid coupler used as a $180^{\circ}$ power divider.

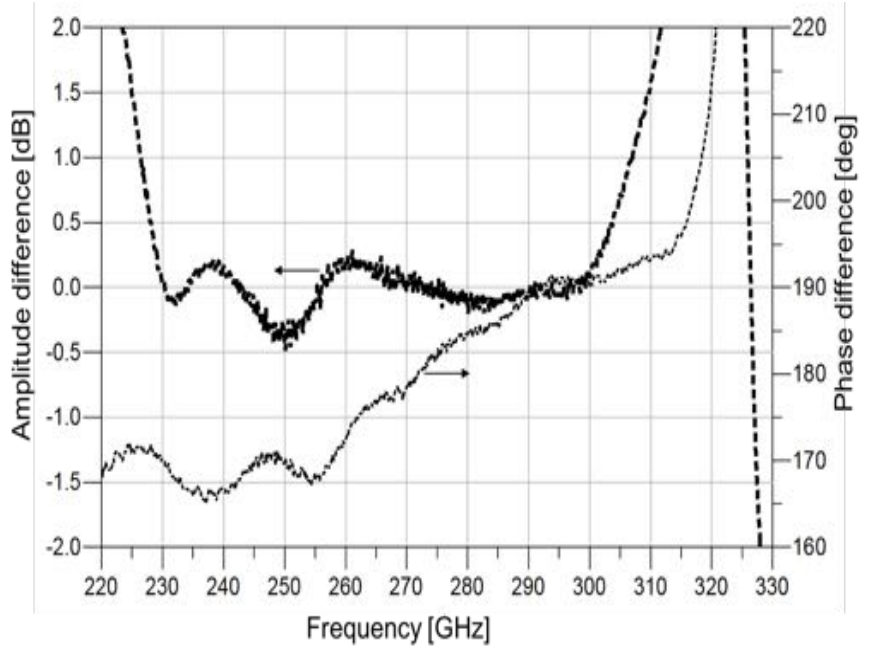

Fig. 5. Measured amplitude and phase differences (imbalances) for the Jband micromachined waveguide hybrid coupler outputs used as a $180^{\circ}$ power divider.

\section{CONCLUSIONS}

A $230-300 \mathrm{GHz}$ silicon micromachined waveguide hybrid coupler fabricated in a double H-plane split waveguide design was presented. Measurements of the hybrid coupler used as a $180^{\circ} 3-\mathrm{dB}$ power divider show coupling coefficients of $3.2 \pm 0.4 \mathrm{~dB}$, an amplitude imbalance better than $-0.5 /+0.3 \mathrm{~dB}$, a phase imbalance better than $-14^{\circ} / 11^{\circ}$ and an input reflection coefficient below $-14 \mathrm{~dB}$. These excellent results indicate a successful use of the developed double H-plane split, low-loss micromachined waveguide technology. The initially measured losses are in fact in parity with what was reported for a similar micromachined coupler operating at $\mathrm{W}$-band that achieved a $10 \%$ smaller relative bandwidth [1].

\section{ACKNOWLEDGMENT}

This work has been funded by the Swedish Foundation for Strategic Research via the Synergy Grant Electronics SE13007.

\section{REFERENCES}

[1] Y. Li, P.L. Kirby, O. Offranc and J. Papapolymerou, "Silicon micromachined $\mathrm{W}$-band hybrid coupler and power divider using DRIE technique," IEEE Microw. Wireless Compon. Lett., vol. 18, no. 1, pp. 22-24, Jan. 2008.

[2] M. Moallem and K. Sarabandi, "A non-contact submillimeter-wave Sparameters measurement technique for multiport micromachined devices," IEEE Trans. Terahertz Science Tech., vol. 4, no. 3, pp. 338346, May 2014.

[3] J.T. Do, Q. Yu, J.L. Hesler and N.S. Barker, "A 330-500 GHz micromachined directional coupler," in IEEE MTT-S Int. Dig., Jun. 2014.

[4] S. Liu, J. Hu, Y. Zhang, L. Li, W. Zhao, R. Xu and Q. Xue, "Silicon micromachined waveguide quadrature-hybrid coupler at terahertz frequency band," J. Infrared Milli Terahz Waves, vol. 36, no. 8, pp. 709719, Aug 2015.

[5] T. J. Reck, C. Jung-Kubiak, J. Gill and G. Chattopadhyay, "Measurements of silicon micromachined waveguide components at 500-750 GHz," IEEE Trans. Terahertz Science Tech., vol. 4, no. 1, Jan 2014 . 
[6] B. Beuerle, U. Shah, and J. Oberhammer, "Low-loss 220-330 GHz micromachined waveguide technology enabled by double H-plane split", unpublished

[7] U. Shah, E. Decrossas, C. Jung-Kubiak, T. Reck, G. Chattopadhyay, I. Mehdi, and J. Oberhammer, "Submillimeter-Wave 3.3-bit RF MEMS
Phase Shifter Integrated in Micromachined Waveguide," IEEE Trans. Terahertz Science and Tech., vol. 6, no. 5, pp. 706-715, Sept. 2016.

[8] B. Beuerle, J. Campion, U. Shah and J. Oberhammer, "Low return loss 220-330 GHz microwave absorbers integrated in micromachined waveguides", unpublished 